DOI 10.1007/s00702-005-0381-6

J Neural Transm (2005) 112: 1687-1694

Journal of _ Neural

Transmission

(C) Springer-Verlag 2005

Printed in Austria

\title{
The North American Multiple System Atrophy Study Group
}

\author{
S. Gilman ${ }^{1}$, S. J. May ${ }^{2,7}$, C. W. Shults ${ }^{3,7}$, C. M. Tanner ${ }^{4}$, \\ W. Kukull ${ }^{5}$, V. M.-Y. Lee ${ }^{6}$, E. Masliah ${ }^{7}$, P. Low ${ }^{8}$, P. Sandroni ${ }^{8}$, \\ J. Q. Trojanowski ${ }^{6}$, L. Ozelius ${ }^{9}$, T. Foroud ${ }^{10}$, \\ and The North American Multiple System Atrophy Study Group

\footnotetext{
${ }^{1}$ Department of Neurology, University of Michigan, Ann Arbor, MI,

${ }^{2}$ Department of Family \& Preventive Medicine, University of California San Diego, La Jolla, CA,

${ }^{3}$ VA San Diego Healthcare System, San Diego, CA,

${ }^{4}$ Department of Clinical Research, Parkinson's Institute, Sunnyvale, CA,

${ }^{5}$ Department of Epidemiology, University of Washington, Seattle, WA,

${ }^{6}$ Department of Pathology \& Laboratory Medicine, Center on Neurological

Disease Research, University of Pennsylvania, Philadelphia, PA,

${ }^{7}$ Department of Neurosciences, University of California, San Diego, La Jolla, CA,

${ }^{8}$ Department of Neurology, Mayo Clinic, Rochester, MA,

${ }^{9}$ Department of Molecular Genetics, Albert Einstein College of Medicine, Bronx, NY, and

${ }^{10}$ Department of Medicine and Molecular Genetics, Indiana University School of Medicine, Indianapolis, IN, USA
}

Received February 12, 2005; accepted September 10, 2005

Summary. The North American Multiple System Atrophy Study Group involves investigators in 12 US medical centers funded by a grant from the National Institutes of Health. The objectives are to examine the environmental and genetic risk factors for MSA; elucidate pathogenic mechanisms underlying the disorder; and refine evaluations used for assessment. During its first year, the group enrolled 87 patients, implemented four cores, and initiated four scientific projects. Most patients among the 87 had parkinsonian features, which frequently began asymmetrically and remained asymmetrical; one-third responded to levodopa and many developed levodopa complications; almost two-thirds of the patients had cerebellar dysfunction, of these $90 \%$ had ataxia; urinary incontinence occurred commonly, and sleep disorders affected most. The investigators studied the effects of oxidative and nitrative stress upon the formation of alpha-synuclein inclusions; generated transgenic models of alpha-synuclein accumulation that recapitulate several behavioral and neuropathological features of MSA; and compared the severity of the autonomic features of MSA, Parkinson's disease and dementia with Lewy bodies. 
Keywords: Multiple system atrophy, parkinsonism, cerebellar ataxia, autonomic failure.

\section{Introduction}

The North American Multiple System Atrophy (MSA) Study Group (NAMSASG) consists of investigators in 12 participating medical centers in the United States. Cliff Shults, M.D. organized the group and obtained funding through a five-year program project grant from the National Institutes of Health, which began on September 30, 2003. The overall specific aims of the program project are to elucidate the risk factors for MSA, both environmental and genetic; study pathogenic mechanisms contributing to the development of MSA; and evaluate and refine clinical and laboratory assessments used to diagnoses and follow the course of the illness. The program project consists of four scientific projects supported by four cores. The principal investigators and co-investigators of the projects include C.M. Tanner, M.D., Ph.D., Parkinson's Institute and W. Kukull, Ph.D., University of Washington (Project 1, Risk Factors for MSA); V.M.-Y. Lee, Ph.D., University of Pennsylvania (Project 2, Mechanisms of Synuclein Pathologies in MSA); C. Shults, M.D. and E. Masliah, M.D., University of California San Diego (Project 3, Alpha-synuclein in Transgenic Models of MSA); and P.A. Low, M.D. and P. Sandroni, M.D., Ph.D., Mayo Clinic (Project 4, Clinical and Laboratory Indices that Differentiate and Predict Outcome in MSA and Parkinson's Disease with Autonomic Failure). The principal investigators and co-investigators of the cores include C. Shults, M.D., University of California San Diego and Sid Gilman, M.D., University of Michigan (Core A, Administrative and Clinical); R. Thomas, Ph.D. and S. May, Ph.D., University of California San Diego (Core B, Data); J. Trojanowski, M.D., Ph.D., University of Pennsylvania (Core C, Neuropathology); and L. Ozelius, Ph.D., Albert Einstein College of Medicine and Tatiana Foroud, Ph.D., Indiana University (Core D, Genetics). In this summary of the NAMSA-SG, we will present briefly a progress report of the first year of our activities.

\section{Results and discussion}

\section{Participating sites}

Table 1 lists the sites participating in the NAMSA-SG and the functions served by these sites. Oversight of the program project comes from Dr. Shults at the University of California San Diego, which contains the Administrative and Data Cores as well as Project 3. Project 1 is performed at the Parkinson Institute. The University of Pennsylvania houses the Neuropathology Core and Project 2; Albert Einstein College of Medicine is home to the Genetics Core; and the Mayo Clinic hosts Project 4. The remaining institutions serve as enrolling sites for patients and control subjects who have no signs or symptoms of MSA. When these institutions enroll subjects, they acquire a minimum data set, which includes the principal features of the disorder (Tables 2-6). Enrollment occurs at 11 of the 12 sites; the Albert Einstein College of Medicine does not enroll subjects. Enrollment and data entry occurs via a secure web-based data entry 
Table 1. Sites participating in the NAMSA-SG and functions served in the sites

- University of California San Diego

(Administrative and Data Cores, Project 3, Enrolling site)

- Parkinson Institute (Project 1, Enrolling site)

- University of Pennsylvania (Neuropathology Core, Project 2, Enrolling site)

- Mayo Clinic (Project 4, Enrolling site)

- Albert Einstein College of Medicine (Genetics Core)

- University of Maryland (Enrolling site)

- University of Rochester (Enrolling site)

- Boston University (Enrolling site)

- University Hospitals of Cleveland (Enrolling site)

- Baylor College of Medicine (Enrolling site)

- University of Michigan (Enrolling site)

- University of Virginia (Enrolling site)

system, which was developed within the first six months of the beginning of grant funding.

\section{MSA patients enrolled during the first year}

Over approximately 12 months, we have enrolled 87 patients with probable MSA. These subjects include 56 male and 31 female subjects with a mean age of $63 \pm 8.6$ years (mean \pm standard deviation). In this group, parkinsonian and autonomic symptoms were the most common presenting features, followed by cerebellar symptoms and combinations of symptoms (Table 2). Examination revealed parkinsonian features in a high percentage of these patients, with bradykinesia, postural instability of the parkinsonian type and rigidity affecting a large majority of subjects (Table 3). Over one-third of these subjects had resting tremor, two-thirds showed postural tremor, and approximately one-third manifested persistent asymmetry. Also, more than half of the patients who received levodopa treatment had a continuing beneficial response. Cerebellar features also affected a majority of these subjects; unsteadiness and falls attributed to cerebellar ataxia were found in most patients, and ataxia of gait, speech and limbs was found in most of these patients (Table 4). Dysphagia was a

Table 2. Type of symptom onset in the first MSA patients enrolled

\begin{tabular}{lcc}
\hline Symptom & n/Total N & Percent of patients \\
\hline Parkinsonian & $26 / 87$ & 29.9 \\
Autonomic & $26 / 87$ & 29.9 \\
Cerebellar & $8 / 87$ & 9.2 \\
Parkinsonian + cerebellar & $5 / 87$ & 5.7 \\
Parkinsonian + autonomic & $6 / 87$ & 6.9 \\
Cerebellar + autonomic & $0 / 87$ & 0 \\
Parkinsonian + cerebellar + autonomic & $2 / 87$ & 2.3 \\
Ambiguous or incomplete information* & $14 / 87$ & 16.1 \\
\hline
\end{tabular}

${ }^{*}$ Due to partially missing dates, the order of symptom onset could not be determined 
S. Gilman et al.

Table 3. Parkinsonian features in the first MSA patients enrolled

\begin{tabular}{lcc}
\hline Feature & $\mathrm{n} /$ Total N & Percent of patients \\
\hline Bradykinesia* $^{*}$ & $82 / 84$ & 97.6 \\
Rigidity* $^{*}$ Postural instability* & $71 / 84$ & 84.5 \\
Resting tremor* & $81 / 84$ & 96.4 \\
Postural tremor* & $33 / 84$ & 39.3 \\
Unilateral onset* & $54 / 84$ & 64.3 \\
Persistent asymmetry* & $34 / 84$ & 40.5 \\
Levodopa response & $29 / 84$ & 34.5 \\
$\quad$ Beneficial response & & \\
No response & $32 / 87$ & 36.8 \\
$\quad$ Not administered & $26 / 87$ & 29.9 \\
$\quad$ Initial but not & $25 / 87$ & 28.7 \\
$\quad$ continuing response & $4 / 87$ & 4.6 \\
Wearing-off** & & \\
On/off** & $17 / 22$ & 77.3 \\
Peak dose dyskinesias** & $5 / 22$ & 22.7 \\
Biphasic dyskinesias** & $6 / 22$ & 27.3 \\
Off-dystonia** & $1 / 22$ & 4.6 \\
\hline
\end{tabular}

* Only for patients with parkinsonism, ${ }^{* *}$ only for patients with motor complications

prominent symptom, affecting about two-thirds of patients. Autonomic dysfunction also affected most subjects, with urinary incontinence and incomplete bladder emptying reported in a large majority, and over one-third of subjects had a history of postural syncope (Table 5). Also, fecal incontinence affected close to one-third of patients. Among the males, erectile dysfunction was very frequent, affecting 52 of the 56 men enrolled. Other prominent features include sleep disorders in approximately two-thirds of patients, vivid dreams in about half, and complaints of memory impairment in almost one-third of subjects (Table 6).

In summary, this data set reveals the following clinical trends: (1) parkinsonian features affect most subjects; (2) these features begin asymmetrically and remain asymmetrical in a substantial number of cases; (3) approximately

Table 4. Cerebellar features in the first MSA patients enrolled

\begin{tabular}{lll}
\hline Feature & $\mathrm{n} /$ Total N & Percent of patients \\
\hline Cerebellar dysfunction & & \\
$\quad$ Gait ataxia* & $48 / 53$ & 90.6 \\
$\quad$ Ataxic dysarthria* & $42 / 53$ & 79.2 \\
$\quad$ Limb ataxia* & $46 / 53$ & 86.8 \\
$\quad$ Sustained nystagmus* & $20 / 53$ & 37.7 \\
Overlap dysfunction & & \\
$\quad$ Dysphagia & $60 / 87$ & 69.0 \\
Dysarthria & $74 / 87$ & 85.1 \\
Unsteadiness with falls & $83 / 87$ & 95.4 \\
\hline
\end{tabular}

* Only for patients with ataxia 
Table 5. Autonomic features in the first MSA patients enrolled

\begin{tabular}{lll}
\hline Feature & $\mathrm{n} /$ Total N & Percent of patients \\
\hline Postural syncope & $36 / 87$ & 41.4 \\
Urinary incontinence & $77 / 87$ & 88.5 \\
Fecal incontinence & $25 / 87$ & 28.7 \\
Incomplete bladder emptying & $70 / 87$ & 80.5 \\
Male erectile dysfunction & $52 / 56$ & 92.9 \\
\hline
\end{tabular}

three quarters of the MSA patients had received levodopa, more than half of these responded to levodopa, and many developed levodopa complications; (4) ataxia appears in more than half of all patients and almost all patients presenting with cerebellar dysfunction; (5) a majority of patients complain of dysphagia; (6) urinary incontinence occurs frequently, much more commonly than postural syncope; (7) about one-third of patients report fecal incontinence; (8) sleep disorders affect about two-thirds of cases; and (9) memory impairment and confusion are frequent complaints.

\section{Research projects}

Project 1 consists of an epidemiological study to investigate the determinants of MSA using a case-control design. The project will investigate the hypotheses that (1) specific occupational or avocational chemical exposure increases the risk of MSA; (2) specific dietary factors directly affect the risk of MSA; (3) exposure to the following can affect the risk of MSA: tobacco, caffeine, alcohol, anti-inflammatory drugs and head trauma; and (4) familial aggregation of MSA or associated neurodegenerative diseases will be found in the families of persons with MSA. During the first year, this project has developed the individual components of detailed lifetime history interviews and begun telephone interviews with 79 subjects.

Project 2 will elucidate the effects of oxidative/nitrative stress upon the formation of filamentous alpha-synuclein inclusions and assess the changes in gene expression in alpha-synuclein aggregate-bearing oligodendrocytes in MSA. The project has already demonstrated that oxidative and nitrative challenges have differential effects on alpha-synuclein fibrillization and may cause alpha-

Table 6. Other features in the first MSA patients enrolled

\begin{tabular}{lcc}
\hline Feature & $\mathrm{n} /$ Total N & Percent of patients \\
\hline Memory impairment & $27 / 87$ & 31.0 \\
Confusion & $15 / 87$ & 17.2 \\
Vivid dreams & $40 / 87$ & 46.0 \\
Sleep disorder & $54 / 87$ & 62.1 \\
Hallucinations & $6 / 87$ & 6.9 \\
(drug-induced) & & \\
Frontal release signs & $15 / 87$ & 17.2 \\
Apraxia & $7 / 87$ & 8.0 \\
\hline
\end{tabular}


synuclein lesions through distinct mechanisms (Norris et al., 2003). The project examined the pathological interactions of tau and alpha-synuclein in MSA and related synucleinopathies, and detected tau and 14-3-3 proteins in subsets of glial cytoplasmic inclusions (GCIs) (Giasson et al., 2003b). These findings suggest that pathological interactions between alpha-synuclein and tau induce tau fibrillization (Giasson et al., 2003a; Lee et al., 2004). In contrast to other neurodegenerative disorders with tau inclusions, GCIs contain predominantly hypophosphorylated species of tau. The project has generated monoclonal antibodies to ubiquitin, synphilin and Parkin because these proteins have been implicated in alpha-synuclein lesions (Murray et al., 2003; Pawlyk et al., 2003; Sympathu et al., 2003). As many GCIs and other alpha-synuclein inclusions were ubiquitin positive with the newly produced anti-ubiquitin monoclonal antibodies, ubiquitination may be important in the pathogenesis of GCIs, but ubiquitination of alpha-synuclein appears to be a downstream event in the pathogenesis of GCIs (Sympathu et al., 2003). The Parkin monoclonal antibodies produced in this project did not stain alpha-synuclein lesions even though these antibodies are specific for Parkin, as shown by screening these antibodies in Parkin knock-out mice and showing that the antibodies do not cross react with other irrelevant proteins (Pawlyk et al., 2003). The synphilin monoclonal antibodies stained a small subset of alpha-synuclein inclusions (Murray et al., 2003). Thus, Parkin and synphilin may not be important in the pathogenesis of alpha-synuclein lesions in MSA, while oxidative/nitrative mechanisms do contribute to GCI formation, albeit by differentially modifying alpha-synuclein.

Project 3 will utilize transgenic models of alpha-synuclein accumulation and aggregation to determine the behavioral, neurochemical and neuropathological effects of alpha-synuclein accumulation and the effects of oxidative stress and of nitration on the accumulation of alpha-synuclein. This project has already created five transgenic mouse lines overexpresssing human alpha-synuclein under the control of the murine myelin basic protein promoter, and these mice recapitulate many of the behavioral and pathological features of MSA.

Project 4 will determine whether clinical and laboratory indices, especially autonomic indices, will differentiate MSA, Parkinson's disease, and Parkinson's disease with autonomic failure and will predict outcome. The project will also undertake mechanistic studies to differentiate these disorders with investigations focused on autonomic regulation. Two studies have been completed, one comparing autonomic deficits in dementia with Lewy bodies (DLB), MSA, and Parkinson's disease (PD), and the other focused on the quality of life in MSA. The first study compared data from 20 DLB patients to 20 MSA and 20 PD patients (Thaisetthawatkul et al., 2004). Orthostatic hypotension was found in $10 / 20 \mathrm{DLB}, 17 / 20 \mathrm{MSA}$, and 1/20 PD patients $(\mathrm{p}=0.023,0.003)$. The results of the Composite Autonomic Scoring Scale (CASS) were 5.2 \pm 2.0 for DLB, $8.1 \pm 1.3$ for MSA, and $2.2 \pm 1.2$ for PD $(\mathrm{p}<0.00001)$. The conclusions from the study are that autonomic dysfunction occurs frequently in DLB and is intermediate in severity between the autonomic disorders of MSA and PD. The second study evaluated life satisfaction and the relationship of disease parameters, physical function, and depressive symptoms to life satisfaction in 98 MSA patients (Benrud-Larson et al., 2005). The study revealed low 
life satisfaction, with a mean of 38.8 on a 100 -point visual analogue scale $(0=$ extremely dissatisfied, $100=$ extremely satisfied $)$. Thirty-five percent of patients reported moderate to severe depressive symptoms on the Beck Depression Inventory (BDI $\geqslant 20)$. Data from the SF-36 Physical Component Summary Scale were approximately 1.5 standard deviations below the mean of a normative sample of healthy adults the same age. Hierarchical regression analysis revealed that disease parameters (autonomic deficits, symptom severity, and symptom duration) accounted for $22 \%$ of the variance in life satisfaction $(\mathrm{p}<0.01)$. Physical function as measured by the SF-36 did not account for any variance beyond the disease parameters; however, depressive symptoms accounted for an additional $15 \%$ of the variance in life satisfaction $(p<0.001)$. These results suggest that among patients with MSA, depressive symptoms may be an important determinant of life satisfaction in addition to disease-related factors and should be assessed routinely. Treatment of comorbid depression may improve quality of life in patients with MSA, despite the other debilitating deficits.

\section{Acknowledgements}

The work is supported by a program project grant from the NIH (P01 NS044233) to CWS. Research at UCSD is supported by The William M. Spencer, Jr. Endowed Fellowship and Research Fund in Multiple System Atrophy.

\section{Member of NAMSA-SG}

\section{Steering committee}

C. W. Shults, M.D., S. Gilman, M.D., C. Tanner, M.D., Ph.D., W. Kukull, Ph.D., V. Lee, Ph.D., E. Masliah., M.D., P. Low, M.D., I. Shoulson, M.D., R. Thomas, Ph.D., S. May, Ph.D., J. Trojanowski, M.D., Ph.D., L. Ozelius, Ph.D. and T. Foroud, Ph.D.

\section{Enrolling sites and investigators}

University of California, San Diego - C. W. Shults, M.D.

Parkinson Institute - C. Tanner, M.D., Ph.D., J. Tetrud, M.D., N. Huang, M.D.

Mayo Clinic, Rochester, MN - P. Low, M.D., P. Sandroni, M.D.

University of Michigan - S. Gilman, M.D.

University Hospitals of Cleveland - T. Chelimsky, M.D., and D. Riley, M.D.

Baylor College of Medicine - J. Jankovic, M.D., and W. Ondo, M.D.

University of Virginia - G. F. Wooten, M.D.

University of Maryland - S. Reich, M.D.

University of Pennsylvania - M. Stern, M.D. and A. Colcher, M.D.

University of Rochester - F. Marshall, M.D.

Boston University - P. Novak, M.D., Ph.D.

\section{References}

Benrud-Larson LM, Sandroni P, Schrag A, Low PA (2005) Depressive symptoms and life satisfaction in patients with multiple system atrophy. Mov Disord 15: 71-75

Giasson BI, Forman MS, Higuchi M, Golbe LI, Graves CL, Kotzbauer PT, Trojanowski JQ, Lee VM (2003a) Initiation and synergistic fibrillization of tau and alpha-synuclein. Science 300: $636-640$

Giasson BI, Mabon ME, Duda JE, Montine TJ, Robertson D, Hurtig HI, Lee VM, Trojanowski JQ (2003b) Tau and 14-3-3 in glial cytoplasmic inclusions of multiple system atrophy. Acta Neuropathol (Berl) 106: 243-250 
Lee VM, Giasson BI, Trojanowski JQ (2004) More than just two peas in a pod: common amyloidogenic properties of tau and alpha-synuclein in neurodegenerative diseases. Trends Neurosci 27: 129-134

Murray IJ, Medford MA, Guan HP, Rueter SM, Trojanowski JQ, Lee VM (2003) Synphilin in normal human brains and in synucleinopathies: studies with new antibodies. Acta Neuropathol (Berl) 105: 177-184

Norris EH, Giasson BI, Ischiropoulos H, Lee VM (2003) Effects of oxidative and nitrative challenges on alpha-synuclein fibrillogenesis involve distinct mechanisms of protein modifications. J Biol Chem 278: 27230-27240

Pawlyk AC, Giasson BI, Sampathu DM, Perez FA, Lim KL, Dawson VL, Dawson TM, Palmiter RD, Trojanowski JQ, Lee VM (2003) Novel monoclonal antibodies demonstrate biochemical variation of brain parkin with age. J Biol Chem 278: 48120-48128

Sampathu DM, Giasson BI, Pawlyk AC, Trojanowski JQ, Lee VM (2003) Ubiquitination of alpha-synuclein is not required for formation of pathological inclusions in alpha-synucleinopathies. Am J Pathol 163: 91-100

Thaisetthawatkul P, Boeve BF, Benarroch EE, Sandroni P, Ferman TJ, Petersen R, Low PA (2004) Autonomic dysfunction in dementia with Lewy bodies. Neurology 62: 1804-1809

Authors' address: Dr. S. Gilman, Department of Neurology, University of Michigan, 300 North Ingalls St. 3D15, Ann Arbor, MI 48109-0489, USA, e-mail: sgilman@umich.edu 